\title{
General practice management of TMDs
}

\author{
Treatment of temporomandibular disorders by stabilising splints in general dental practice: results after initial \\ treatment R. W. Wassell, N. Adams and P. J. Kelly Br Dent J 2004; 197: 35-41
}

Introduction Little is known about how effective general dental practitioners (GDPs) are in treating temporomandibular disorders (TMD). The overall aim of this study was to compare the lower stabilising splint (SS) with a non-occluding control (CS) for the management of TMD in general dental practice.

Method A total of 93 TMD patients attending 11 GDPs were randomly allocated to SS or CS. Diagnosis was according to International Headache Society Criteria. Outcome criteria included pain visual analogue scale (VAS), number of tender muscles, aggregate joint tenderness, inter-incisal opening, TMJ clicks and headaches. Splints were fitted one week after baseline and patients were followed-up every three weeks to three months; those not responding to CS after six weeks (< 50\% VAS reduction) were crossed over to SS for a further 3 months.

Results Documentation was returned from nine GDPs for 72 patients ( 38 for SS, 34 for CS). At six weeks, mean improvements were noted for all outcome criteria, but less so for clicking. There were no significant differences between splints $\left[\chi^{2}\right]$. Seventeen CS patients had $<50 \%$ VAS reduction and were provided with SS in the cross-over group. CS patients with $>50 \%$ VAS reduction were significantly younger than CS patients who crossed-over (ANOVA, $p=0.009$ ) and had significantly less diagnoses of TMJ clicking $\left(\chi^{2}, p<0.05\right)$. At the conclusion of the trial 16 patients were referred for specialist management: 11 non-responders $(<$ 50\% VAS reduction), one of whom needed occlusal adjustment and five responders also needing occlusal adjustment.

Conclusions At six weeks SS gave similar relief to CS for all outcome criteria. Patients who crossed-over from CS to SS were more likely to be older and have clicking TMJs. At the end of treatment nine of 11 non-responders to SS had a diagnosis of disc displacement with reduction. However, 80\% TMD patients were managed effectively by GDPs using splints for periods of up to five months.

\section{IN BRIEF}

- During the first six weeks of treatment, the occlusal surface of the splint made no significant contribution to any of the outcomes measured suggesting that the occlusion is a relatively unimportant factor influencing recovery in the majority of TMD patients seen in practice.

- A small proportion of patients needed treatment for up to five months to obtain a satisfactory response. These were the patients who crossed over from the non-occluding control splint to the stabilising splint. They tended to be older with TMJ clicking.

- Clicking was not especially responsive to treatment with the stabilising splint, but discomfort was reduced in three quarters of patients with clicking TMJs. It was difficult to make a reliable diagnosis of disc displacement with reduction using the trial criteria.

- Suitably trained and interested GDPs can manage four out of five TMD patients in general practice; a link with specialist services is recommended to deal with non-responding patients.

\section{COMMENT}

It has been asserted, possibly through mis-information but possibly because of entrepreneurial considerations that 'It is a service [provision of interocclusal appliances] that only the dentist can render. It is a golden opportunity for dentistry:. ${ }^{1}$ Could the legacy of such sentiments underpin the mantra of treating TMD with splint therapy?

So how refreshing it is to learn from a group of mainly general dental practitioners, within the constraints of their methodology, some of which were imposed by the moral imperative, that 'sham' splints had the same favourable outcome as those that covered the occlusal surfaces.

Two other findings deserve comment:

1. Referral for occlusal adjustment was carried out on one nonresponder and 5 responding patients. Is this treatment approach at variance from the conclusions of a recent systematic review ${ }^{2}$ that state 'There is an absence of evidence, from RCTs, that occlusal adjustment treats (or prevents) TMD'? It may be that although there is no evidence of the efficacy of occlusal adjustment, it does not necessarily mean that this is not effective for some selected patients.

2. This study has shown that general dental practitioners can successfully treat patients with TMD. The caveat is that they had undergone postgraduate training in the care of such patients. Indeed the assurance and skill that these dentists would have inevitably conveyed to their patients as shown, for example, by the meticulous and time-consuming chairside method that was used to refine the stabilising splints may have contributed to an 'added' placebo effect.

Returning to the key finding of this study that 'sham splints' did every bit as well as those that cover the occlusal surfaces, should we now not be asking ourselves if the provision of these former splints be our first line of treatment for those with TMD?

\section{J. R. Radford, Senior Clinical Lecturer,} Unit of Restorative Dental Care and Clinical Dental Sciences, University of Dundee, Dundee Dental Hospital and School doi:10.1038/sj.bdj.4811417

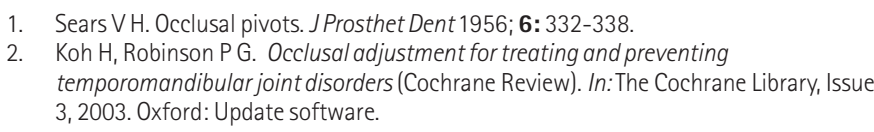

